\title{
Sputtering yield measurements at glancing incidence using a quartz crystal microbalance
}

\author{
Robert D. Kolasinski ${ }^{a}$ \\ Department of Mechanical Engineering, California Institute of Technology, 1200 E. California Blvd., \\ Pasadena, California 91125 \\ James E. Polk, Dan Goebel, and Lee K. Johnson \\ Propulsion and Materials Engineering Section, NASA Jet Propulsion Laboratory, 4800 Oak Grove Dr., \\ Pasadena, California 91109
}

(Received 3 November 2006; accepted 28 December 2006; published 26 January 2007)

\begin{abstract}
Low energy sputtering yields at grazing incidence have been investigated experimentally using a quartz crystal microbalance (QCM) technique. This method involved precoating the QCM with a thin film of the desired target material and relating the resonance frequency shift directly to mass loss during ion bombardment. A highly focused, low divergence ion beam provided a well defined incidence angle. Focusing most of the ion current on the center of the target allowed for higher sensitivity by taking into account the radial mass sensitivity of the QCM. Measurements of $\mathrm{Mo}, \mathrm{Cu}$, and $\mathrm{W}$ sputtering yields were taken for low energy $(80-1000 \mathrm{eV}) \mathrm{Xe}^{+}$and $\mathrm{Ar}^{+}$to validate this experimental method. The target films ranged from 3.5 to $8.0 \mu \mathrm{m}$ in thickness and were deposited so that their crystal structure and density would match those of the bulk material as closely as possible. These properties were characterized using a combination of scanning electron microscope imagery, profilometry, and x-ray diffraction. At normal incidence, the sputtering yields demonstrated satisfactory agreement with previously published work. At angles of incidence up to $40^{\circ}$ off normal, the data agreed well with predictions from existing theoretical models. Sputtering yields were found to increase by a factor of 1.6 over this range. The optimum angle for sputtering occurred at $55^{\circ}$, after which the yields rapidly decreased. Measurements were taken up to $80^{\circ}$ from the surface normal. (C) 2007 American Vacuum Society. [DOI: 10.1116/1.2435375]
\end{abstract}

\section{INTRODUCTION}

Ion beam etching of surfaces has become an important fabrication process for a wide range of industrial applications. The ability to create surface structures smaller than $1 \mu \mathrm{m}$ in width has been applied by the electronics industry to the fabrication of integrated circuits ${ }^{1}$ and hard disk drive heads. ${ }^{2}$ Because ion etching occurs along one direction only, greater control over the surface structure shape may be achieved compared to chemical processes where the etching is isotropic. For this reason, the effect of the beam angle of incidence on surface sputtering is an important consideration for this application, as it may be used in conjunction with different masking techniques to create desirable surface structures. Greater processing capability may also be gained by taking advantage of higher material removal rates at larger angles of incidence. Increased sputtering yields at larger angles of incidence also lead to faceting of sharp edges, which may be an undesirable effect.

Sputtering effects from ion beams are also important in a number of applications in the aerospace industry. Ion thrusters used by NASA for high efficiency spacecraft propulsion are susceptible to sputter damage. ${ }^{3,4}$ These thrusters (which are similar to Kaufman style ion sources) extract ions through a set of molybdenum grids. A small fraction of these

${ }^{a)}$ Present address: Sandia National Laboratories, P. O. Box 969, MS 916, Livermore, CA 94551; electronic mail: rkolasi@sandia.gov ions undergo charge exchange collisions with neutral propellant atoms, creating slow moving ions which strike the grid apertures at varying angles of incidence. While these charge exchange ions are of fairly low energy $(150-500 \mathrm{eV})$, the sputter damage caused by this process accumulates over time and reduces thruster propellant throughput capability. Recent work by Williams et al. ${ }^{5}$ Tartz et al., ${ }^{6}$ and Yalin et al. ${ }^{7}$ has focused on sputtering processes relevant to a variety of spacecraft materials.

Because of the importance of sputtering to surface science and materials research, a large number of published studies contain yield measurements, many of which are reviewed in Ref. 8. The effect of incidence angle on the sputtering of metallic materials has been investigated extensively by Bay and Bohdansky for moderate energy (2-10 keV), light ions.' The present study focuses on measurement techniques for low energy, heavy ion sputtering at near grazing incidence, an area which has not received extensive treatment in previous work. In prior studies, the most common methods for determining material removal rate were by weight loss ${ }^{10}$ or a measurement of thickness change. ${ }^{11}$ Absolute sputtering yields at low bombarding energies tend to be more difficult to measure because of the increased testing time required to obtain a detectable mass loss. Transient effects such as ion source drift and changes in surface roughness increase the potential for measurement error in such cases. Such factors may have contributed to the noticeable spread in sputtering 
data at low energies. For example, yields measured in different laboratories for $\mathrm{Xe}^{+} \rightarrow \mathrm{Mo}$ vary by a factor of 5 at $100 \mathrm{eV}$.

The sputtering yields reported here have been measured using a quartz crystal microbalance (QCM). This technique has a high mass sensitivity (on the order of $5 \times 10^{-9} \mathrm{~g}$ ) and allows measurements to be made in situ. This enables a large database of measurements to be accumulated in a relatively short period of time. The general process involves precoating the QCM with a thin film of a material of interest and subsequently bombarding it with ions. Use of a beam rather than a plasma discharge is necessary in order to investigate angle of incidence effects.

QCMs have been previously employed by Anderson and Bay to measure normal incidence sputtering yields of metallic films using high energy ions. ${ }^{12}$ In the present study, this method was modified to allow for measurements at nonnormal incidence. A narrow, highly collimated beam was used to provide a well defined incidence angle. Because the beam did not impinge on the entire target surface, the radial mass sensitivity of the QCM was taken into account to allow for changes in the irradiation area with different angles of incidence. Etching of the target due to energetic neutrals created by charge exchange processes could potentially introduce a systematic bias in measured sputtering yields. In this study, this effect was measured in situ by the QCM by deflecting all charged species off the target electrostatically. In addition, a low profile mounting scheme allows the beam to be angled almost parallel to the target for glancing angle measurements. A Peltier cooler attached to the crystal eliminated resonance frequency drift due to thermal variations while enabling both translation and rotation of the target in vacuum.

This article begins with a brief review of sputtering theory at varying incidence angles. A section discussing the experimental hardware follows and includes details of the quartz crystal microbalance method, experimental facility, and diagnostics. Methods used to characterize the target films are presented. The correction for the radial dependence of the QCM mass sensitivity is discussed, along with potential error sources. Normal incidence sputtering yields obtained for Mo, $\mathrm{Cu}$, and $\mathrm{W}$ are compared with previously published data as a validation of the experimental method. A presentation of yield measurements obtained at varying angles of incidence concludes the article.

\section{THEORY}

During a typical low energy collision process, a projectile atom penetrates a surface until it reaches a depth where it has transferred most of its kinetic energy to other target atoms. An atom near the surface is sputtered if enough energy is transferred as a result of collisions with the projectile or recoiling target atoms. As the angle of incidence $\theta$ (defined with respect to the surface normal) increases, more energy from the projectile is deposited near the surface. The probability of an atom gaining sufficient energy to leave the surface is higher as a result, and the sputtering yield $Y$ increases.
Above $\theta=45^{\circ}$, surface effects become more important, and prior experimental work shows that $Y$ achieves a maximum for values of $\theta$ between $50^{\circ}$ and $80^{\circ}$ and decreases rapidly thereafter. It has been suggested that this decrease is due to increased scattering of incident ions off of the surface as well as surface roughness effects. ${ }^{8}$

Sigmund published a comprehensive theoretical treatment of sputtering in 1969 (Ref. 13) where he applied the Boltzmann transport equation to an amorphous, infinite target. His theory predicts the following relationship between the sputtering yield at normal incidence and at an angle $\theta$ with respect to normal:

$$
Y(\theta)=Y(0) /(\cos \theta)^{f} .
$$

The value of the exponent $f$ may be calculated analytically and depends weakly on projectile mass and energy, as well as target mass. For most ion-target combinations, taking $f$ to be $5 / 3$ is an accurate approximation.

Sigmund's model predicts normal incidence sputtering yields to within a factor of 2 (and in many cases, within $40 \%$ ) of reported experimental values. A number of limitations arise due to assumptions included in the model which break down for incident angles greater than $45^{\circ}$. Equation (1) is unable to predict the subsequent drop off in $Y$ at glancing angles, a fact which has been attributed to the simplified treatment of surface effects necessary in order to obtain an analytical solution to the Boltzmann equation. ${ }^{14}$

An empirical model has been proposed by Yamamura to predict the variation of $Y$ with $\theta .^{15}$ Reasonable agreement with reported experimental results has been achieved for high energy light ions. The lack of quality experimental data for heavy ions, however, has inhibited the development of empirical models for such cases. Monte Carlo programs such as TRIM (transport of ions in matter) (Ref. 16) provide more realistic estimates of sputtering yields at higher incidence angles, although large differences between $Y$ values obtained with these simulations and experiments have been noted. ${ }^{17}$

\section{EXPERIMENTAL HARDWARE}

In the present article, sputtering yield measurements were obtained using a quartz crystal microbalance. As an ion beam bombards the thin film, atoms are ejected from the surface and the resonance frequency of the QCM changes. For a given change in areal mass density $\sigma$, the shift in frequency $v$ will be given by $\Delta \sigma / \sigma_{o}=\Delta v / v_{o}$. This expression is valid only for small mass changes, but its useful range can be extended significantly by using more sophisticated relationships between frequency change and mass loss. ${ }^{18}$ A disadvantage associated with this technique is that the physical properties and sputtering behavior of thin films deposited on the QCM surface may not resemble the bulk material. To address this concern, a detailed characterization of the thin films was undertaken using a number of surface analysis techniques. Attention has been given to an accurate characterization of the ion flux to the target as well as a detailed analysis of potential error sources. These efforts are discussed in detail in the following sections. 


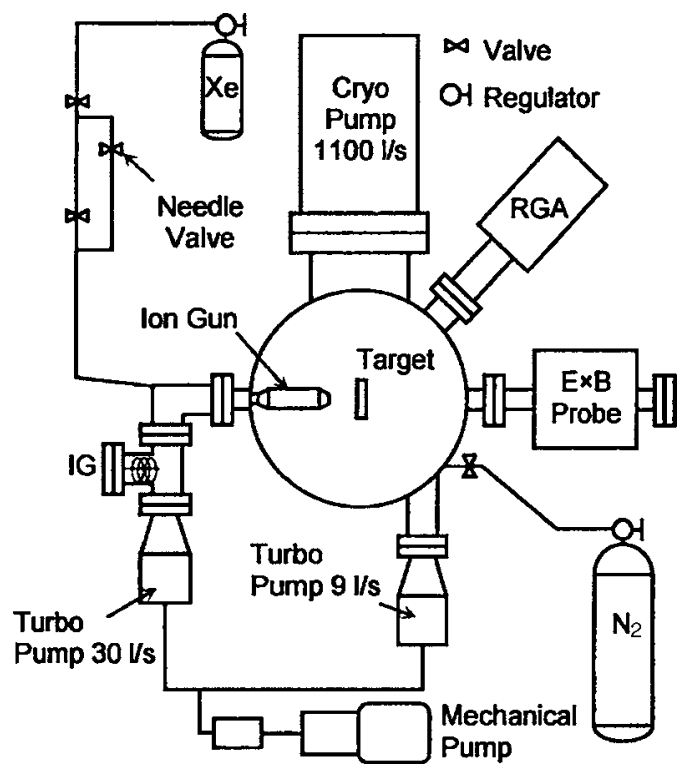

FIG. 1. Sputtering facility schematic.

\section{A. Vacuum facility}

Minimizing the arrival rate of reactive gas components to the target surface necessitated the use of an ultrahigh vacuum (UHV) facility. In this case, the vacuum chamber used was capable of reaching a base pressure of approximately 1.1 $\times 10^{-7} \mathrm{~Pa}\left(8.0 \times 10^{-10}\right.$ Torr $)$. A schematic of the sputtering facility is shown in Fig. 1. The stainless steel chamber measured approximately $0.6 \mathrm{~m}$ in length with a $0.3 \mathrm{~m}$ diameter, and the interior surfaces were electropolished to eliminate virtual leaks which increase pump-down time. Two small turbo pumps initially evacuated the main chamber and differential pumping port to a pressure of $0.1 \mathrm{~Pa}\left(10^{-3}\right.$ Torr $)$ and a $20 \mathrm{~cm}$ (8 in.) cryogenic pump subsequently reduced the pressure to $6.7 \times 10^{-6} \mathrm{~Pa}\left(5 \times 10^{-8}\right.$ Torr $)$. The cryogenic pump also had the advantage of a large xenon pumping speed (approximately $1000 \mathrm{l} / \mathrm{s}$ ), allowing a low working pressure to be established during ion gun operation. An ion pump provided an additional $300 \mathrm{l} / \mathrm{s}$ of pumping speed. After a bakeout at $200{ }^{\circ} \mathrm{C}$ for $12 \mathrm{~h}$, the final base pressure was achieved. To reduce contamination of the system, the chamber was pressurized with nitrogen gas after each set of experiments was completed.

Two ionization gauges were used to measure the pressures in the main chamber and differential pumping port. A residual gas analyzer (RGA) with a mass range of $200 \mathrm{amu}$ characterized the background gas composition. The RGA included an electron multiplier which allowed residual gas components to be detected at partial pressures as low as $1.3 \times 10^{-9} \mathrm{~Pa}\left(10^{-12}\right.$ Torr $)$. The most common residual gas components included $\mathrm{CO}$ at a partial pressure of 4 $\times 10^{-8} \mathrm{~Pa}\left(3 \times 10^{-10}\right.$ Torr $)$ and $\mathrm{H}_{2} \mathrm{O}$ at $1.3 \times 10^{-8} \mathrm{~Pa}(1$ $\times 10^{-10}$ Torr). The ion source used in this study was capable of delivering a current to the target such that the ratio of ion to background gas flux was on the order of 30:1. This indicates that the vacuum conditions and ion beam strength were

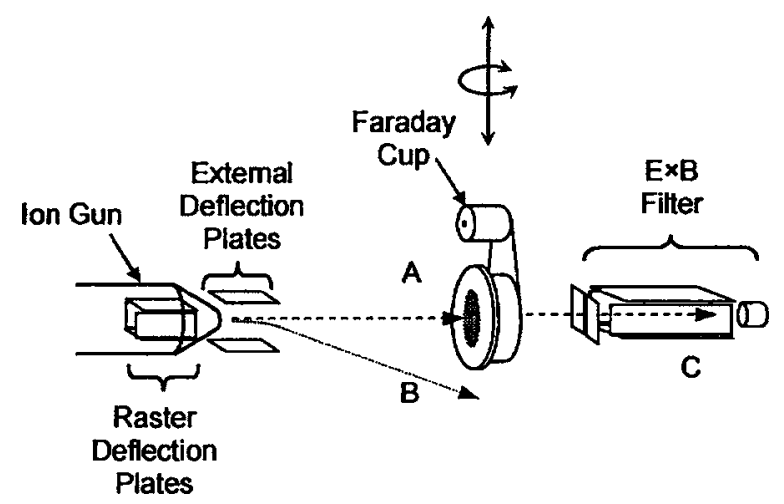

FIG. 2. Ion beam diagnostics.

sufficient to not only maintain a dynamically clean surface during bombardment but also to ensure that the target could be initially sputter cleaned for tests at lower energies.

\section{B. QCM}

Commercially available AT-cut QCMs were used throughout this investigation. When unloaded, the crystals have a resonance frequency of $6 \mathrm{MHz}$. The crystals were unpolished and were coated with a thin gold layer. Depending on the range of incidence angles being investigated, two different QCM mounting schemes were used. For $\theta<50^{\circ}$, a conventional crystal holder with a graphite shield was used with a thermoelectric cooler attached to the base. In this configuration, the QCM could be cooled or warmed to within $0.25{ }^{\circ} \mathrm{C}$ of the desired set point temperature using a computer controlled loop. In addition, use of the cooler allowed for rotation of the target in situ, which would not have been possible with liquid cooling lines.

For angles of incidence greater than $50^{\circ}$, the front shield of the holder began to shadow the face of the QCM. This resulted in an underestimation of the etch rate, thereby biasing the measurement of $Y$ lower. To circumvent this problem, an alternate mounting scheme was employed for glancing incidence. In this configuration, the crystal was bonded to three supports using an UHV rated conducting silver epoxy. The low profile of the supports allowed the beam to be aligned almost parallel to the QCM surface. The total current to the target from the beam could be measured directly in this configuration. Since there was no way to attach a Peltier cooler to this assembly, the temperature of the sample could not be directly controlled.

\section{Ion source}

An accurate determination of the bombardment conditions at the target is often a complicated process in practice. In general, the ion beam must be well defined in terms of ion energy and species, a condition often achieved by mass separating the beam. ${ }^{19}$ This involves using a series of filters to remove unwanted charge states and energies from the beam upstream of the target. For the experiments reported here, an alternate approach allowed for implementation in a smaller vacuum facility. This arrangement is shown in Fig. 2. Neutral 
$\mathrm{Xe}$ or Ar gas was admitted through a needle valve to the differential pumping port of a Kimball Physics ILG-2C low energy ion gun. The gas is ionized with a filament cathode, extracted, and focused through a series of electrostatic lenses. Near the exit aperture, a set of deflection plates rasters the beam to more evenly distribute the ion dose to the QCM target mounted approximately $40 \mathrm{~mm}$ downstream. This is the nominal test configuration and is indicated by case A in Fig. 2. The incident ion current is measured with a Faraday cup mounted a fixed distance below the target. A profile of beam current density is generated by scanning the QCM/Faraday probe assembly across the beam by means of a linear translation stage. While the vacuum facility base pressure is typically $6.7 \times 10^{-4} \mathrm{~Pa}\left(5 \times 10^{-6}\right.$ Torr $)$ during ion source operation, higher pressures inside the gun cause a large amount of charge exchange (CEX) ions to be generated upstream of the exit aperture. The Faraday probe traces allow only the total charge incident on the target to be measured; additional techniques are necessary to account for high energy neutrals formed in CEX reactions. A second set of deflection plates just downstream of the gun exit was used to deflect ions away from the QCM, as shown in case B. CEX neutrals are unaffected by this process, and the QCM is used to measure the erosion from this species in situ. The QCM and Faraday cup can be retracted completely to allow the beam to pass into an $E \times B$ probe. Located just downstream of the target, this instrument characterizes the double ion content of the beam. Because double ions could not be separated upstream of the target, the discharge parameters of the gun are adjusted to minimize the quantity of $\mathrm{Xe}^{2+}$ and $\mathrm{Ar}^{2+}$ present.

An impinging high energy ion beam causes the ejection of secondary electrons from a surface, which makes ion beam current measurements problematic. Emitted secondary electron current cannot be distinguished from arriving ion current. The Faraday probe used in this study was designed to minimize this effect. The current collecting cup was fabricated from molybdenum, which has a low secondary electron emission coefficient. To prevent the escape of secondary electrons, a high aspect ratio geometry for the collector cup was used (approximately 4:1). The collector was housed within a $6.35 \mathrm{~mm}(0.25 \mathrm{in}$.) diameter stainless steel shield. The shield was biased $18 \mathrm{~V}$ below ground potential to repel electrons created by other instruments in the vacuum facility and to provide an additional potential barrier for the escape of emitted secondary electrons within the probe.

The small diameter of the ion beam $(5-6 \mathrm{~mm})$ introduced a number of challenges for accurate current measurement and probe alignment. The size of the Faraday probe shield's knife edge aperture was chosen to be $0.9 \mathrm{~mm}$ based on the need to balance signal strength with resolution. The narrow beam width also demanded precise probe and target positioning. Using welded bellows segments in the alignment fixture allowed the sample to be rotated and translated along one axis and small adjustments to be made along the other two

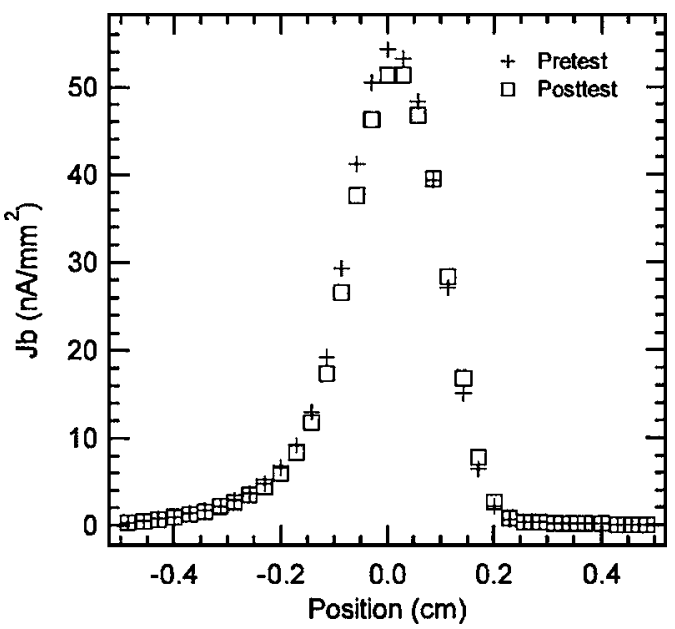

FIG. 3. Pretest and post-test beam current density profile for $300 \mathrm{eV} \mathrm{Xe}$ ions.

axes. A micrometer gimbal was attached to the lower bellows while the upper bellows was attached to a linear translation stage equipped with a stepper motor.

The ion source operating parameters were selected to maximize the ion current to the target while minimizing the double ion fraction and maintaining an axisymmetric beam. A typical beam current density profile is shown in Fig. 3. Measurements taken approximately $1 \mathrm{~h}$ apart are overlaid to illustrate the beam current drift experienced during a typical test run. Although a series of electrostatic lenses within the ion source allowed some control over the focusing of the beam, an $x-y$ raster was applied to create a more uniform dose over the target surface. This created a rectangular pattern over the target surface which changed with time (the periods of the $x$ and $y$ scans were not synchronized). The sweep frequencies were much larger than the response of the picoammeter used to measure the current signal detected by the probe. For the ion energies used in this study, the peak beam current density ranged from 23 to $92 \mathrm{nA} / \mathrm{mm}^{2}$. With rastering, the beam diameter based on the full width at half maximum could be varied between 1.4 and $4 \mathrm{~mm}$.

In this study, the ion beam was assumed to be axisymmetric and the $j_{b}$ profiles measured with the Faraday probe along one axis were used for calculations of the sputtering yields. To verify beam axisymmetry, the probe was centered on the beam at the point of maximum current density and then the beam was deflected in a gridlike pattern over the probe using the raster deflection plates, effectively creating a twodimensional map. At high voltages the deflection distorts the profile (making it inadequate for use as a quantitative measurement). However, qualitative information on beam axisymmetry is obtained from this method and may be used to determine how representative the $j_{b}$ profile is of the overall beam. The beam map displayed in Fig. 4 was taken during the same test as the $j_{b}$ profiles displayed by Fig. 3 .

An $E \times B$ probe accounted for double ions and other impurities in the beam. The same probe originally constructed for Ref. 20 was also employed here. In this study, Duchemin 


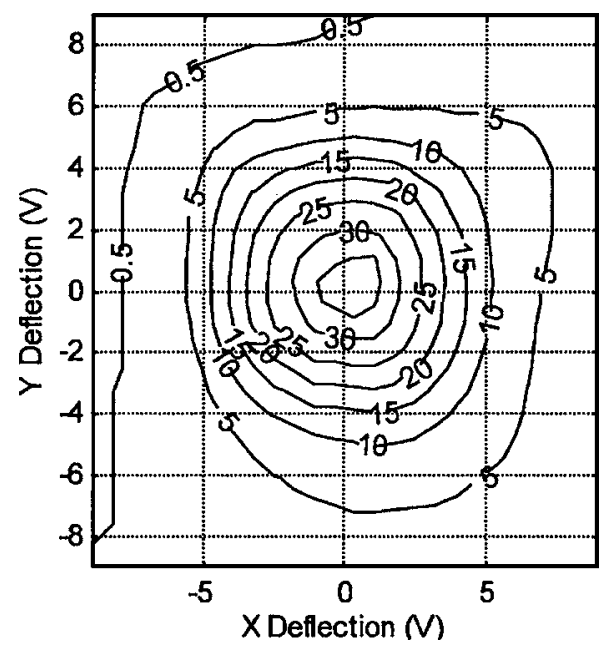

FIG. 4. Constant current lines (nA) for a $300 \mathrm{eV} \mathrm{Xe}$ ion beam, mapped as a function of deflection voltage.

and Polk found that the double ion fraction in the beam could be minimized to negligible levels by lowering the ion source discharge voltage as much as possible. Probe measurements verified that for the $40 \mathrm{~V}$ discharge used in the present work, the ratio of $\mathrm{Xe}^{2+}$ to $\mathrm{Xe}^{+}$was less than $4 \times 10^{-4}$.

\section{TARGET CHARACTERIZATION}

In the present study, three target materials $(\mathrm{Mo}, \mathrm{Cu}$, and $\mathrm{W})$ were investigated and deposited on QCMs in the form of thin films. The molybdenum coatings were deposited using a magnetron sputtering process developed by Duchemin and Polk. ${ }^{20}$ The thicknesses of these coatings were determined through profilometry and were found to be $3.5 \mu \mathrm{m}$. The copper and tungsten films were also generated by magnetron sputtering and were coated to thicknesses of 9.0 and $5.0 \mu \mathrm{m}$, respectively. The parameters for these depositions are given in Table I.

In practical applications, bulk materials are the sputtering targets, so the physical properties of the coatings used in this experiment should be representative of the bulk material. Two techniques have been employed to examine the film properties in detail, including scanning electron microscope (SEM) imagery and x-ray diffraction. Because of its use in electric propulsion for sputter resistant components, the Mo films were of primary interest here, and the $\mathrm{Cu}$ and $\mathrm{W}$ coatings were not characterized as extensively. The SEM and $\mathrm{x}$-ray diffraction analysis results are presented for the Mo films only.

TABLE I. Cu and $\mathrm{W}$ film deposition parameters.

$$
\begin{gathered}
\text { Deposition rate } 110 \AA / \mathrm{min} \\
\text { Working gas Argon } \\
\text { Pressure } 1.3 \mathrm{~Pa} \\
\text { Bias voltage } 0 \mathrm{~V}
\end{gathered}
$$

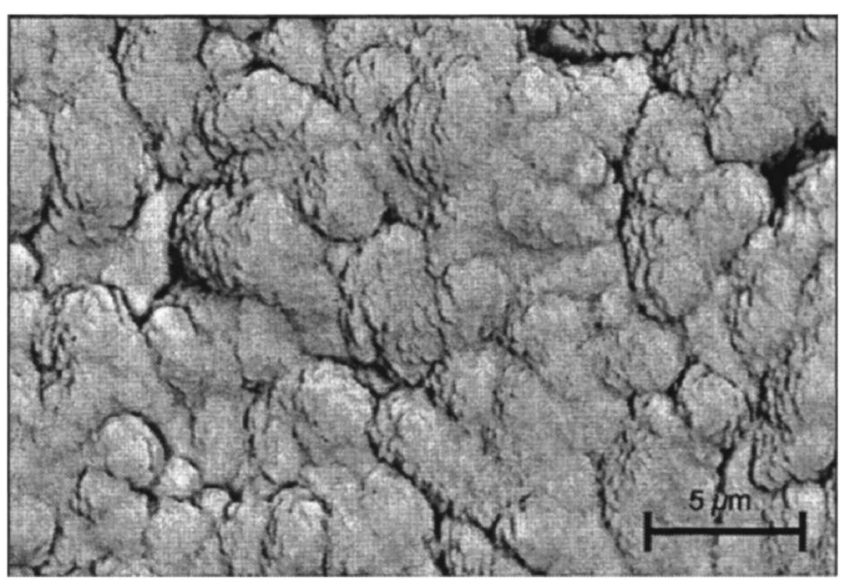

FIG. 5. SEM image revealing surface topography of a Mo film.

\section{A. SEM}

A scanning electron microscope provided insight into the surface topography and chemical composition of the deposited films. Figure 5 displays the surface, whereas Fig. 6 shows a cross section of a crystal broken in liquid nitrogen. In both cases, the film was imaged using electrons backscattered from the sample. The images reveal that the surface is significantly textured with visible clusters of crystals between 3 and $5 \mu \mathrm{m}$ in length. The $3.5 \mu \mathrm{m}$ thick layer of molybdenum can be seen deposited on the gold coated quartz crystal in the cross section image. (The gold layer is approximately $200 \mathrm{~nm}$ thick.) The grain structure appears to be roughly columnar and discontinuities in the film appear to coincide with surface irregularities in the quartz substrate.

Energy dispersive x-ray (EDX) analysis was used to detect impurities in the coating surface. While not the most precise means of accomplishing this, EDX does allow qualitative verification that impurity levels are, in fact, small. For the coatings discussed here, no traces of substances other than Mo were detected. Images from the SEM also did not reveal any large concentrations of impurities, suggesting that

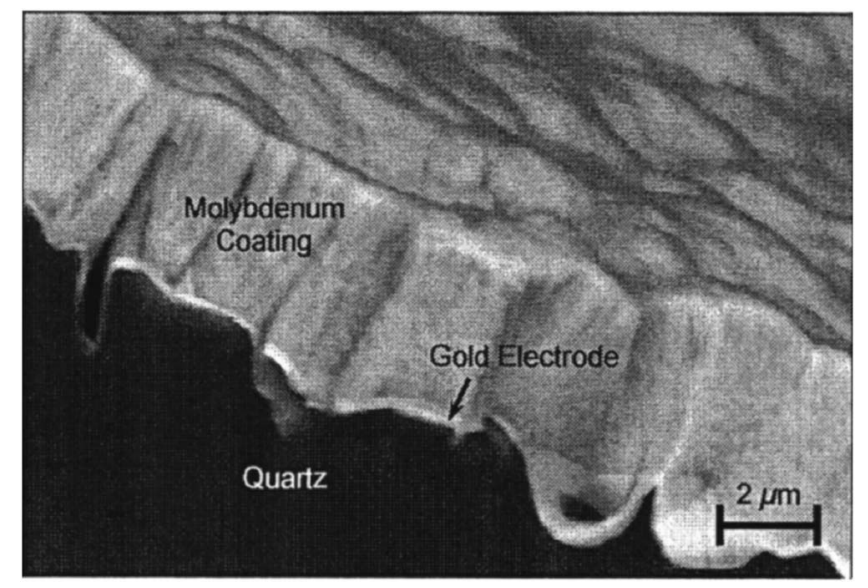

FIG. 6. SEM image of a cross section of a crystal fractured in liquid nitrogen. 


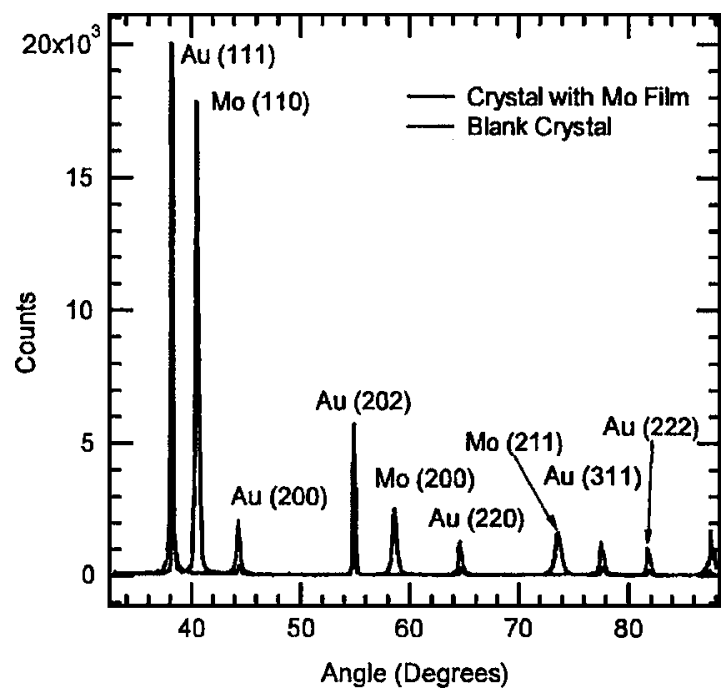

FIG. 7. X-ray diffraction scans of Mo coated and blank QCMs.

any impurities were scattered diffusely throughout the coating and were not present in quantities above the detection threshold.

\section{B. X-ray diffraction}

The crystal structure of the target material could potentially influence the measured sputtering yields. The effect of angle of incidence on single crystal structures is particularly dramatic, as certain angles allow significant channeling into the surface, thereby reducing the sputtering yield. ${ }^{21}$ Given the potential influence of crystal structure on yield measurements, the lattice parameters and the density of the deposited thin films were calculated using $\mathrm{x}$-ray diffractometer measurements.

Interpretation of the diffraction data reported here is complicated by the presence of peaks from both the Au electrode and the Mo film. Scans of both a blank and a molybdenum coated QCM were taken and are displayed in Fig. 7. In the scan of the crystal blank, only the Au peaks are visible and display a pattern characteristic of a face centered cubic crystal structure. In the scan of the coated crystal, three additional peaks corresponding to the Mo layer appear. Their spacing indicates a body centered cubic structure, the same as bulk molybdenum. The peaks corresponding to each element are labeled in Fig. 7 along with the Miller indices of the crystal plane responsible for each reflection.

The atomic spacing may be calculated directly from the diffraction scan. The copper electrode $\mathrm{x}$-ray tube used in this study had a characteristic radiation wavelength of $\lambda$ $=1.54178 \AA$. Based on this wavelength and the location of the peaks, the atomic spacing in the film was calculated to be $3.149 \AA$, less than $0.08 \%$ larger than the bulk material. This would correspond to a coating density of $\rho_{E}=10.20 \mathrm{~g} / \mathrm{cm}^{3}$, less than $0.3 \%$ lower than the bulk value.

\section{Summary}

The observations from the SEM and x-ray diffraction analysis support the conclusion that the thin films are a reasonable approximation to the bulk materials. The coatings have the same crystal structure as the bulk material, and the interatomic spacing indicated by the $\mathrm{x}$-ray diffraction measurements indicates a comparable film density. The EDX analysis shows low levels of impurities in the deposited films as well. The coatings appear to be composed of columnar grains, which are smaller than the average bulk material size. However, since the grain diameters of polycrystalline metals is still small compared with the irradiation area, one would expect this difference to have a very minor effect on the sputtering behavior.

\section{EXPERIMENTAL PROCEDURE}

Each crystal used was cleaned with chemical solvents and rinsed with alcohol and de-ionized water prior to inserting it into the experimental facility. Once the desired facility base pressure had been achieved, gas was admitted to the differential pumping port of the ion gun. The typical operating pressure of this device was on the order of $2.6 \times 10^{-2} \mathrm{~Pa} \mathrm{(2}$ $\times 10^{-4}$ Torr). A $3 \mathrm{~h}$ stabilization period was allowed after the gun was activated.

The Faraday probe was centered on the beam by adjusting its position with the micrometer gimbal stage and stepper motor until the current measured by the probe was maximized. A beam map was taken to confirm that the rastering created an axisymmetric beam current distribution. Once the probe alignment was completed, a beam current density profile was taken, and the QCM was moved into the beam. The sample temperature, differential pumping port pressure, and QCM monitor output were recorded with a computer-based data acquisition system. In each case, the sample was etched to a depth of at least $40 \AA$, after which the ion beam was deflected completely off of the crystal surface to measure the sputter rate contribution from energetic neutrals.

The output from the thickness monitor for the case of $1 \mathrm{keV} \mathrm{Xe}+$ ions incident on Mo is shown in Fig. 8. The total etch rate is the initial slope, which is simply the superposition of the effects of the ions and neutrals incident on the surface of the QCM. The etch rate due to neutrals is measured by the QCM in situ and subtracted from the total erosion rate to obtain the contribution of the ions only.

For measurements with bombarding energies less than $200 \mathrm{eV}$, it was possible for the residual gas components in the facility to form a protective layer on the QCM surface which could not be destroyed by the low energy ions. For these cases, the surface was sputter cleaned at $1 \mathrm{keV}$ immediately prior to etching. The effect of this phenomenon on the measurements is further discussed in Sec. VI. In addition, for measurements at different angles of incidence, the surface was preconditioned by etching at normal incidence to a depth of $80 \AA$. This ensured that the initial surface topography of the sample was similar for all measurements.

Even though numerous steps had been taken to ensure the stable operation of the ion source, a small amount of drift 


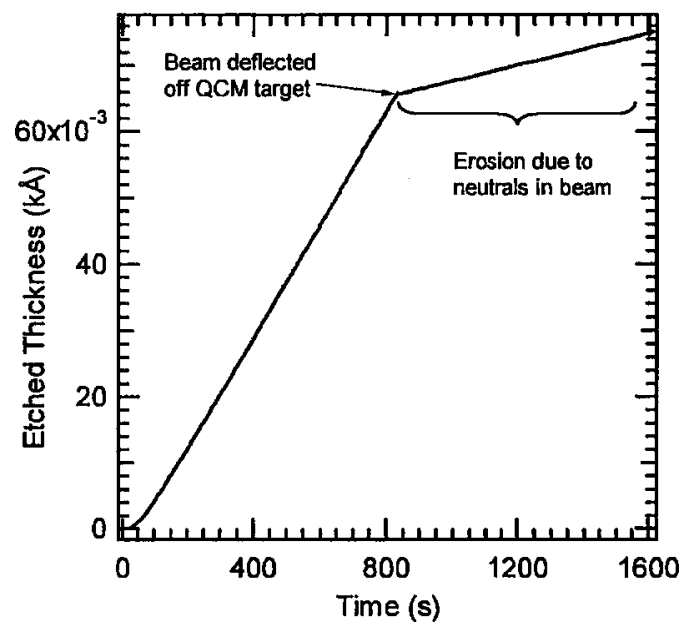

FIG. 8. Measured Mo film thickness change during bombardment by $1 \mathrm{keV}$ $\mathrm{Xe}^{+}$ions.

(typically $5 \%$ or less) in the current output was unavoidable. To characterize the magnitude of this variation and its effect on the sputtering yield measurement, a second $j_{b}$ profile was taken at the conclusion of each test. For measurements taken at normal incidence and for $\theta<45^{\circ}$, this was the only method of determining the total drift. In the sputtering yield calculations, a monotonic variation between the pretest and post-test profile was assumed. The QCM mount used for glancing incidence measurements allowed the total current to the target to be monitored continuously throughout the etching process. During these tests, the $j_{b}$ variation appeared to be coupled with ambient temperature variations. Because the test duration was short compared to the temperature drift time scale, the $j_{b}$ variations were monotonic, validating the assumption used in the near-normal incidence measurements.

As previously mentioned, a major advantage of this technique is the large number of measurements which can be taken in a short period of time. Typically, five test runs at $1 \mathrm{keV}$ can be conducted during a single day of operation. As one might expect, the testing time required at lower energies is substantially longer. A single $80 \mathrm{eV}$ molybdenum test requires nearly $17 \mathrm{~h}$ to complete.

\section{A. Data analysis}

The indicated thickness change represents an average over the entire QCM surface. Because the removal of mass from the target surface was not uniform, a correction was necessary to account for the variations in QCM sensitivity as a function of radius $r$ and polar angle $\varphi$. An analytical function for this sensitivity $c_{f}$ was derived by Cumpson and Seah: $:^{22}$

$$
c_{f}(r, \varphi)=c_{o} \exp \left[-r^{2}\left(\alpha_{1} \cos ^{2} \varphi+\beta_{1} \sin ^{2} \varphi\right)\right] .
$$

The constants $\alpha_{1}, \beta_{1}$, and $c_{o}$ depend on the material properties of quartz as well as the crystal geometry according to

$$
\begin{aligned}
& \alpha_{1}^{2}=\frac{\pi^{2} \bar{C}_{66}}{8 R h_{0}^{3} M_{1}}\left(1-\frac{8 k_{26}^{2}}{\pi^{2}}-\frac{2 \rho_{E} h_{E}}{\rho_{Q} h_{0}}\right), \\
& \beta_{1}^{2}=\frac{\pi^{2} \bar{C}_{66}}{8 R h_{0}^{3} C_{55}}\left(1-\frac{8 k_{26}^{2}}{\pi^{2}}-\frac{2 \rho_{E} h_{E}}{\rho_{Q} h_{0}}\right) .
\end{aligned}
$$

In the above expressions, $C_{55}, C_{66}, M_{1}$, and $k_{26}$ are the elastic constants of quartz, $h_{o}$ and $R$ are the half thickness and radius of curvature of the QCM, and $\rho_{Q}$ is the density of quartz. Numerical values of these constants may be found in Refs. 20 and 22. The film thickness and density are denoted by $\rho_{E}$ and $h_{E}$. At first glance, the dependence of the sensitivity function on $\varphi$ appears counterintuitive, given the circular geometry of the quartz resonator. However, the material properties of the quartz itself are anisotropic $\left(\alpha_{1} \neq \beta_{1}\right)$. The dependence of the response function on $\varphi$ is not strong. Lines of constant $c_{f}$ would form a set of ellipses with the major axis being approximately $12.5 \%$ longer than the minor axis and oriented along the $x$ direction.

Duchemin and Polk pointed out the benefits of using the higher sensitivity of the QCM near the center of the electrode in previous work ${ }^{20}$ and developed a method of applying the expression for $c_{f}$ to sputtering calculations. In particular, if the beam current density profile $j_{b}(r)$ is known, the sputtering yield may be determined by

$$
Y=\frac{\Delta \tau_{\text {meas }} N q \int_{0}^{a} \int_{0}^{2 \pi} c_{f}(r, \varphi) r d r d \varphi}{\Delta t \int_{0}^{a} \int_{0}^{2 \pi} c_{f}(r, \varphi) j_{b}(r) r d r d \varphi} .
$$

Note that $\Delta \tau_{\text {meas }}$ is the average thickness change calculated from the measured resonance frequency shift of the QCM, and $\Delta t$ is the time interval over which the test occurred. In addition, $N$ refers to the target number density and $q$ is the electronic charge. The above expression works well at normal incidence, when the ion dose forms a circular pattern independent of $\varphi$ on the target. For any off normal angle $\theta$, the beam projects an ellipse on the target surface. In this situation, $j_{b}$ becomes a function of $r$ and $\varphi$, with the major axis having been stretched by a factor of $1 / \cos \theta$. The Faraday probe measured the current density profile along a single axis at normal incidence. To modify this profile for cases where the target was angled with respect to the beam, a simple geometrical correction was used. In addition, because the beam is projected over a larger area, the current density decreases. Hence, at glancing angles, measurements are more susceptible to error due to lower current densities.

The Faraday probe averages the measured ion beam current over its entire aperture. Because the aperture size is appreciably comparable to the beam diameter, a slight (but non-negligible) distortion of the $j_{b}$ profile occurs. Retrieving the original profile based on the measured data is not a trivial process, since the averaging process necessarily implies a loss of information over the measurement area of the probe. Given this difficulty, it is assumed that the distortion of the profile due to the probe is small and that the original may be 
obtained by a linear transformation of the measured profile. An iterative scheme was employed to calculate the mapping parameters. Emphasis is placed on minimizing the errors closest to the center of the QCM, near the region of highest sensitivity.

\section{B. Error analysis}

The error of the sputtering yield measurements in this work depends to a significant degree on how accurately the beam current profile has been measured. To better ascertain the effectiveness of the $j_{b}$ profile corrections discussed in the previous section, the QCM was replaced with a carbon disk mounted in the target holder. The disk was isolated from the holder with a thin Nomex® ring and was connected to ground through a picoammeter. The total current to the target was compared with the integrated profile over the QCM surface. In cases where the rastering amplitudes were adjusted to create an axisymmetric beam, measured beam current typically agreed with the total integrated current to within $4 \%$. For cases where the beam was not axisymmetric, the two measurements differed by $10 \%$ or more.

The two raster frequencies $(500$ and $10 \mathrm{~Hz})$ were higher than the picoammeter response time. As a result, the instrument provided an averaged current output. To verify that the filtering process was not improperly biasing the results, a digital oscilloscope was used to capture the signal from the Faraday cup collector over several raster sequences. The average current value measured by the oscilloscope agreed to within $1 \%-2 \%$ of the picoammeter output.

Despite efforts to minimize ion current drift, some small transient effects were unavoidable during etching. The stability of the ion source depended mainly on pressure variations in the differential pumping port and ambient temperature conditions and can be inferred from beam current density profiles before and after each test run as well as from changes in the sputtering rate. The difference in the yield using both the pretest and post-test profiles was calculated to assess the total contribution from this error source. In most cases, the yields calculated from both profiles differed by less than $5 \%$. In the test configuration for glancing angle measurements, the ion current to the target was measured directly. As expected, transient effects were more severe for the longer, lower energy tests.

The QCM positioning uncertainty also contributed to the error. A $0.2 \mathrm{~mm}$ initial alignment uncertainty was assumed for all measurements in this study, corresponding to the step size used in the Faraday probe scans. For measurements where the target was angled with respect to the beam, a $\pm 0.25^{\circ}$ uncertainty in the incidence angle was assumed based on the resolution of the rotary positioning stage.

Temperature changes cause drift in the QCM resonance frequency and introduce systematic errors in the measurements. However, because the ion beam used in this experiment only produced a small dose to the target surface, in most cases the frequency drift was dominated more by ambient temperature changes than from beam heating. The shift in resonance frequency was calculated using curves from
TABLE II. Normal incidence yield measurements.

\begin{tabular}{cccc}
\hline \hline & \multicolumn{3}{c}{$\begin{array}{c}Y \\
\text { (atoms/ion) }\end{array}$} \\
\cline { 2 - 4 } $\begin{array}{c}\text { Energy } \\
(\mathrm{eV})\end{array}$ & $\mathrm{Xe}^{+} \rightarrow \mathrm{Mo}$ & $\mathrm{Ar}^{+} \rightarrow \mathrm{Cu}$ & $\mathrm{Ar}^{+} \rightarrow \mathrm{W}$ \\
\hline 1000 & $1.73 \pm 0.07$ & $3.35 \pm 0.15$ & $\ldots$ \\
700 & $1.25 \pm 0.07$ & $\ldots$ & $\ldots$ \\
600 & $1.12 \pm 0.05$ & $\ldots$ & $0.648 \pm 0.069$ \\
500 & $0.890 \pm 0.043$ & $\ldots$ & $\cdots$ \\
400 & $0.754 \pm 0.042$ & $1.85 \pm 0.08$ & $0.545 \pm 0.026$ \\
300 & $0.550 \pm 0.022$ & $1.50 \pm 0.08$ & $0.475 \pm 0.031$ \\
250 & $0.431 \pm 0.026$ & $\cdots$ & $\cdots$ \\
200 & $0.314 \pm 0.015$ & $0.961 \pm 0.045$ & $0.269 \pm 0.037$ \\
150 & $0.184_{-0.019}^{+0.012}$ & $\cdots$ & $\cdots$ \\
100 & $0.064_{-0.004}^{+0.003}$ & $0.370_{-0.021}^{+0.020}$ & $0.070 \pm 0.009$ \\
80 & $0.023_{-0.001}^{+0.001}$ & $0.238 \pm 0.012$ & $0.026 \pm 0.003$ \\
\hline \hline
\end{tabular}

Ref. 23. During most short duration tests, the thermoelectric cooler regulated the target temperature to within $\pm 0.2{ }^{\circ} \mathrm{C}$ and to within $\pm 2.0^{\circ} \mathrm{C}$ for the longer, lower energy tests. For the glancing angle measurements, the QCM mounting scheme did not allow for the use of a Peltier cooler to regulate the target temperature. The most severe temperature drift experienced with this configuration was less than $2{ }^{\circ} \mathrm{C}$.

The presence of double ions or other impurities in the beam could also potentially bias the results. As previously discussed, the ion source operating parameters were selected to minimize the fraction of double ions in the beam. For a $40 \mathrm{~V}$ discharge, less than $0.04 \%$ of the ions in the beam were $\mathrm{Xe}^{2+}$. Assuming this ratio for an $80 \mathrm{eV}$ ion beam (which represents the worst case), the measured sputtering yield would be biased less than $0.3 \%$ high by the presence of double ions.

Using standard error propagation methods, the error analysis suggests that on average, sputtering yields measured in this article could be considered accurate to within 5\%-6\% at higher energies and between $6 \%$ and $10 \%$ at lower energies.

\section{RESULTS}

As an initial step in validating the experimental setup, the energy dependence of normal incidence sputtering yields was compared with other published values. The ion-target combinations investigated were $\mathrm{Xe}^{+} \rightarrow \mathrm{Mo}, \mathrm{Ar}^{+} \rightarrow \mathrm{Cu}$, and $\mathrm{Ar}^{+} \rightarrow \mathrm{W}$. Values for these normal incidence yields are given in Table II for an energy range of $80-1000 \mathrm{eV}$. Figures 9 and 10 provide a comparison with previously published data from Refs. 6, 7, and 24-31. An empirical fit to these data developed by Matsunami et al. for normal incidence sputtering yields is also included for comparison. ${ }^{32}$ The empirical curve fit of Matsunami et al. slightly underpredicts the trend displayed by most of the published results. It should be noted that this source provides generalized curves designed to fit numerous projectile/target combinations over a wide range of energies and does not necessarily provide a best fit for the 


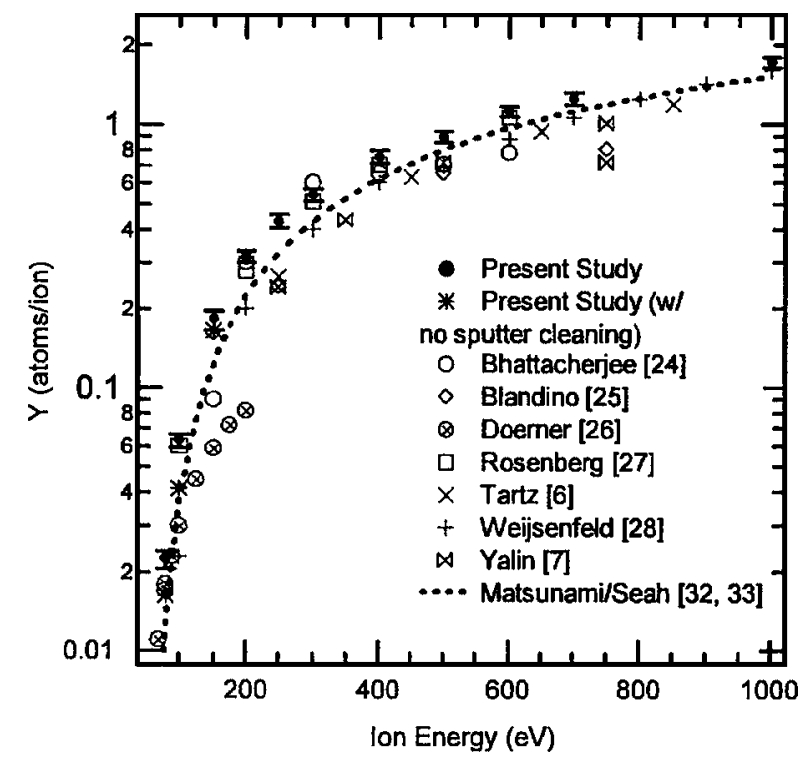

FIG. 9. $\mathrm{Xe}^{+} \rightarrow$ Mo normal incidence sputtering yield measurements for $80-1000 \mathrm{eV}$

specific conditions of interest here. A small adjustment to the curves suggested by Seah for noble gases eliminates this discrepancy. ${ }^{33}$

Also included in the molybdenum data set are yield measurements taken without prior sputter cleaning of the target surface from 80 to $200 \mathrm{eV}$. At low energies, initial sputter cleaning using a higher energy beam had a significant impact on the measured sputtering yield. The inability of the low energy beam to completely remove absorbed monolayers of surface contaminants resulted in a non-negligible level of contaminant coverage. This effect became less pronounced at higher energies, until it was negligible above $200 \mathrm{eV}$. Below $80 \mathrm{eV}$, a clean surface could not be maintained even with prior high energy sputter etching. In addition, the CEX cross sections for $\mathrm{Xe}$ and Ar become larger at lower energies, and at $80 \mathrm{eV}$ fast moving neutrals created by charge exchange account for more than $90 \%$ of the energetic particle flux to the target surface. Distinguishing this effect from erosion

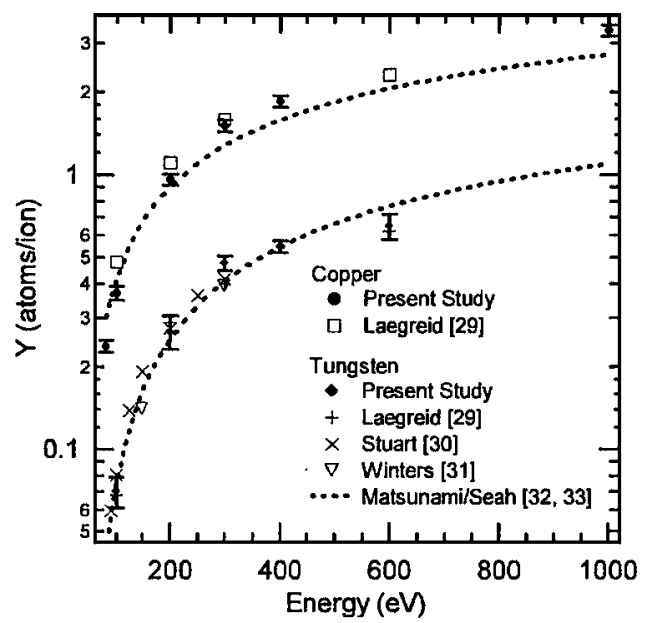

FIG. 10. $\mathrm{Cu}$ and $\mathrm{W}$ sputtering yield measurements for $\mathrm{Ar}^{+}$ion bombardment at normal incidence.
TABLE III. Angular dependence of $\mathrm{Xe}^{+} \rightarrow$ Mo sputtering yields.

\begin{tabular}{cccc}
\hline \hline & \multicolumn{3}{c}{$\begin{array}{c}Y \\
\text { (atoms/ion) }\end{array}$} \\
$\begin{array}{cccc}\theta \\
(\mathrm{deg})\end{array}$ & $300 \mathrm{eV}$ & $500 \mathrm{eV}$ & $1 \mathrm{keV}$ \\
\cline { 2 - 4 } 0 & $0.550 \pm 0.022$ & $0.890 \pm 0.043$ & $1.73 \pm 0.07$ \\
10 & $0.556 \pm 0.023$ & $0.934 \pm 0.052$ & $1.77 \pm 0.08$ \\
20 & $0.651 \pm 0.027$ & $1.02 \pm 0.07$ & $1.91 \pm 0.08$ \\
25 & $0.688 \pm 0.029$ & $1.06 \pm 0.08$ & $1.99 \pm 0.08$ \\
30 & $0.736 \pm 0.032$ & $1.19 \pm 0.06$ & $2.17 \pm 0.09$ \\
35 & $0.789 \pm 0.032$ & $1.24 \pm 0.06$ & $2.34 \pm 0.10$ \\
& & $1.31_{-0.078}^{+0.076}$ & $2.34 \pm 0.10$ \\
40 & $0.831 \pm 0.036$ & $1.41 \pm 0.07$ & $2.51 \pm 0.11$ \\
45 & $0.926 \pm 0.045$ & $1.53 \pm 0.10$ & $2.62 \pm 0.11$ \\
& & & $2.66 \pm 0.14$ \\
50 & $0.920 \pm 0.040$ & $1.53 \pm 0.09$ & $2.80 \pm 0.12$ \\
& $1.02 \pm 0.06$ & $1.60 \pm 0.08$ & \\
55 & $0.948 \pm 0.041$ & $1.61 \pm 0.09$ & $2.77 \pm 0.18$ \\
& $0.931 \pm 0.084$ & $1.58 \pm 0.08$ & \\
60 & $0.934 \pm 0.056$ & $1.56 \pm 0.08$ & $2.72 \pm 0.12$ \\
65 & $0.842_{-0.068}^{+0.066}$ & $1.41 \pm 0.07$ & $2.64 \pm 0.12$ \\
70 & $0.711 \pm 0.043$ & $1.22 \pm 0.07$ & $2.39 \pm 0.11$ \\
75 & $\cdots$ & $1.05 \pm 0.07$ & $2.14 \pm 0.12$ \\
80 & $\cdots$ & $0.842_{-0.061}^{+0.058}$ & $1.87 \pm 0.15$ \\
\hline \hline & $\ldots$ & & \\
\hline
\end{tabular}

from ions becomes increasingly difficult at low energies. The combination of these two effects suggests that the low energy limit of the present experimental setup is approximately $80 \mathrm{eV}$.

$\mathrm{The} \mathrm{Ar}^{+} \rightarrow \mathrm{Cu}$ and $\mathrm{Ar}^{+} \rightarrow \mathrm{W}$ measurements reported here agree well with previously published normal incidence data. At higher energies, the $\mathrm{Xe}^{+} \rightarrow$ Mo data are 5\% higher than the midrange of the literature values and are within the scatter between measurements from different laboratories.

With the accuracy of the experiment established for normal incidence, the dependence of the yields on angle of incidence was then examined for 1000,500 , and $300 \mathrm{eV} \mathrm{Xe} \mathrm{Xe}^{+}$ incident on Mo. The yields for incidence angles between $0^{\circ}$ and $80^{\circ}$ are given in Table III and Fig. 11. The $\cos ^{-f}(\theta)$ dependence predicted by Sigmund anchored to the experimental data at $\theta=0^{\circ}$ is also included in Fig. 11 with the suggested value of $f=5 / 3$ for each energy. For several cases, multiple values for $Y$ are included to establish the measurement repeatability and to validate the error analysis. The dependence of $Y$ on angle of incidence appears to conform to the behavior predicted by Sigmund at low angles. From Fig. 11 , the rate of increase in $Y$ with $\theta$ agrees well with that predicted by Eq. (1) up to $40^{\circ}-45^{\circ}$. A drop-off in this rate is evident until a maximum is achieved between $50^{\circ}$ and $55^{\circ}$. The yields decrease thereafter, with $Y\left(\theta_{\max }\right) / Y(0)$ between 1.6 and 1.8 .

The ratio $Y\left(\theta_{\max }\right) / Y(0)$ and the value of $\theta_{\max }$ determined here are comparable with those of heavy-ion-metallic-target combinations characterized by Oechsner. ${ }^{34}$ However, the optimum angle for maximum sputtering observed in this study appears at a lower value of $\theta$ than previously reported values 


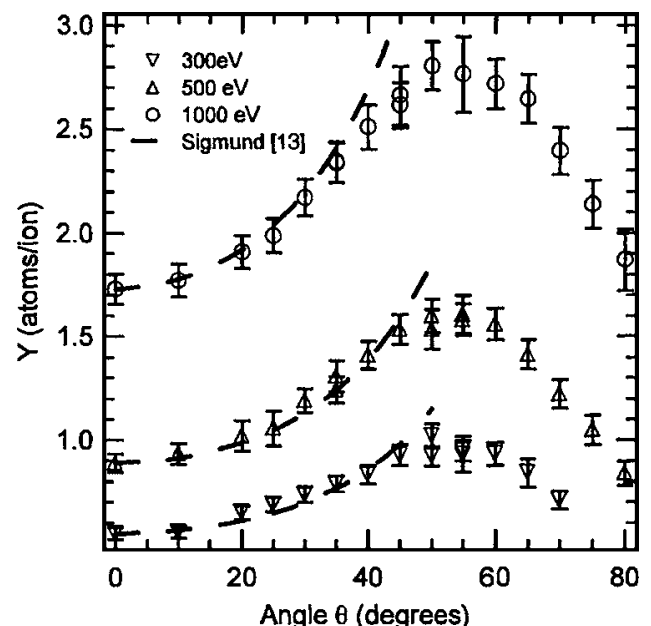

FIG. 11. Sputtering yield variation for $\mathrm{Xe}^{+} \rightarrow \mathrm{Mo}$ as a function of angle of incidence.

at higher energies and lighter atoms. ${ }^{9}$ Such differences are not unexpected, however, given the sizable difference in mass of the incident ions and the larger penetration depths at higher energies. Sputtering yields of materials are known to be influenced by how the energy from the incident ion is deposited within the target medium. ${ }^{13}$ With this in mind, it seems reasonable that differences in $\mathrm{H}^{+}$versus $\mathrm{Xe}^{+}$scattering near the surface can strongly influence the sputtering behavior as a function of angle of incidence, although a more in-depth analysis would be required to verify this assertion.

\section{CONCLUDING REMARKS}

An experimental technique was developed to measure low energy sputtering yields in situ at glancing incidence. A Faraday cup characterized the ion dose to the target, and an $E$ $\times B$ probe quantified the presence of unwanted species in the beam. A measurement of the erosion rate due to energetic neutrals was made by electrostatically deflecting the ions away from the target and subsequently recording the etch rate. Accounting for the radial mass dependence of the QCM allowed a narrow beam to be used. The target thin films were found to be a good approximation to their respective bulk materials using a number of surface analysis techniques.

Yield measurements for $\mathrm{Mo}, \mathrm{Cu}$, and $\mathrm{W}$ targets over an $80-1000 \mathrm{eV}$ energy range were found to be in excellent agreement with published data. Both $\mathrm{Xe}$ and $\mathrm{Ar}$ ion beams were used for bombardment. For $\theta$ ranging between $0^{\circ}$ and $80^{\circ}$, yields for Mo were measured for $\mathrm{Xe}^{+}$energies of $300 \mathrm{eV}, 500 \mathrm{eV}$, and $1 \mathrm{keV}$. An optimum sputtering angle occurred at $50^{\circ}-55^{\circ}$ for these conditions, with $Y\left(\theta_{\max }\right) / Y(0)$ ranging from 1.6 to 1.8. The variation in $Y$ agreed well with the $\cos ^{-f}(\theta)$ dependence predicted by Sigmund for angles less than $45^{\circ}$.

The high sensitivity of the QCM lends itself well to sputtering measurements, allowing etch rates to be measured in situ. The method offers the ability to generate a substantial yield database in a relatively short period of time. With minimal modification, this technique can be applied to a variety of other materials with the restriction that they must be deposited as a thin film at low temperature. How closely these thin films resemble the materials they are intended to simulate remains the most questionable aspect of this experimental method and must be addressed through careful analysis.

\section{ACKNOWLEDGMENTS}

The research described in this article was conducted in part at the Jet Propulsion Laboratory, California Institute of Technology, and was sponsored by NASA. The authors wish to thank a number of individuals at JPL for their assistance with this project, including Ray Swindlehurst and Allison Owens for numerous helpful discussions on the design and fabrication of the diagnostics and UHV equipment used in this study. In addition, the authors express their appreciation to James Kulleck of JPL for obtaining the SEM images and X-ray diffraction scans of the coated QCM targets.

${ }^{1}$ R. Lee, J. Vac. Sci. Technol. 16, 164 (1979).

${ }^{2}$ R. Hsiao, IBM J. Res. Dev. 43, 89 (1999).

${ }^{3}$ R. D. Kolasinski and J. E. Polk, J. Propul. Power 20, 992 (2004).

${ }^{4}$ J. E. Polk, J. R. Anderson, J. R. Brophy, V. K. Rawlin, M. J. Patterson, J. Sovey, and J. Hamley, AIAA Paper No. 99-2446, 1999.

${ }^{5}$ J. D. Williams, M. L. Johnson, and D. D. Williams, AIAA Paper No. 2004-3788, 2004.

${ }^{6}$ M. Tartz, H. Newmann, B. Fritsche, H. J. Leiter, and J. Esch, AIAA Paper No. 2004-4114, 2004.

${ }^{7}$ A. P. Yalin, J. D. Williams, V. Surla, J. Wolf, and K. A. Zoerb, AIAA Paper No. 2006-4336, 2006.

${ }^{8}$ V. S. Smentkowski, Prog. Surf. Sci. 64, 1 (2000).

${ }^{9}$ H. L. Bay and J. Bohdansky, Appl. Phys. 19, 421 (1979).

${ }^{10}$ K. B. Cheney and E. T. Pitkin, J. Appl. Phys. 36, 3542 (1965).

${ }^{11}$ G. Wehner, J. Appl. Phys. 30, 1762 (1959).

${ }^{12}$ H. H. Anderson and H. L. Bay, Radiat. Eff. 13, 67 (1972).

${ }^{13}$ P. Sigmund, Phys. Rev. 184, 383 (1969).

${ }^{14}$ M. Stepanova and S. K. Dew, J. Appl. Phys. 92, 1699 (2002).

${ }^{15}$ Y. Yamamura, Radiat. Eff. 80, 57 (1984).

${ }^{16}$ J. E. Ziegler, J. P. Biersack, and U. Littmark, The Stopping and Range of Ions in Solids (Pergamon, Oxford, 1985), Vol. 1.

${ }^{17}$ R. D. Kolasinski, AIAA Paper No. 2004-4110, 2004.

${ }^{18}$ C. S. Lu and O. Lewis, J. Appl. Phys. 43, 4385 (1972).

${ }^{19}$ H. H. Anderson and H. L. Bay, in Sputtering by Particle Bombardment I: Physical Sputtering of Single-Element Solids, edited by R. Behrisch (Springer, Berlin, 1981), pp. 145-218.

${ }^{20}$ O. B. Duchemin and J. E. Polk, AIAA Paper No. 1999-2858, 1999.

${ }^{21}$ H. E. Roosendaal, in Sputtering by Particle Bombardment I: Physical Sputtering of Single-Element Solids, edited by R. Behrisch (Springer, Berlin, 1981), pp. 219-256.

${ }^{22}$ P. J. Cumpson and M. P. Seah, Meas. Sci. Technol. 1, 544 (1990).

${ }^{23}$ E. P. EerNisse, J. Vac. Sci. Technol. 12, 564 (1975).

${ }^{24}$ S. Bhattacharjee, J. Zhang, V. Shutthanadan, N. R. Shivaparan, and R. J. Smith, Nucl. Instrum. Methods Phys. Res. B 129, 123 (1997).

${ }^{25}$ J. J. Blandino, D. G. Goodwin, and C. E. Garner, Diamond Relat. Mater. 9, 1992 (2000).

${ }^{26}$ R. P. Doerner, D. G. Whyte, and D. M. Goebel, J. Appl. Phys. 93, 5816 (2003).

${ }^{27}$ D. Rosenberg and G. K. Wehner, J. Appl. Phys. 33, 1842 (1962).

${ }^{28} \mathrm{C}$. H. Weijsenfeld, A. Hoogendoorn, and M. Koedam, Physica (Amsterdam) 27, 763 (1961).

${ }^{29}$ N. Laegreid and G. K. Wehner, J. Appl. Phys. 32, 365 (1961).

${ }^{30}$ R. V. Stuart and G. K. Wehner, J. Appl. Phys. 33, 2345 (1962).

${ }^{31}$ H. F. Winters and D. Horne, Phys. Rev. B 10, 55 (1974).

${ }^{32}$ N. Matsunami, Y. Yamamura, Y. Itikawa, N. Itoh, Y. Kazumata, S. Miyagawa, K. Morita, R. Shimizu, and H. Tawara, At. Data Nucl. Data Tables 31, 1 (1984).

${ }^{33}$ M. P. Seah, Nucl. Instrum. Methods Phys. Res. B 229, 348 (2005).

${ }^{34}$ H. Oechsner, Appl. Phys. 8, 185 (1975). 